\title{
Unusual Behaviors of Electric-field Control of Magnetism in Multiferroic Heterostructures via Multifactor Cooperation
}

Ce Feng, ${ }^{\dagger,+}$ Yan Liu, ${ }^{\dagger, t, \perp}$ Haoliang Huang, ${ }^{\S}$ Zhaozhao Zhu, $\|$ Yuanjun Yang, ${ }^{\#}$ You Ba,,$^{\dagger, t}$ Shuying Yan, "Jianwang Cai," Yalin Lu, ${ }^{\S}$ Jinxing Zhang, "Sen Zhang, ${ }^{* \nabla}$ and Yonggang Zhao *,t,

Department of Physics and State Key Laboratory of Low-Dimensional Quantum Physics, Tsinghua University, Beijing 100084, China

Collaborative Innovation Center of Quantum Matter, Beijing 100084, China

$\$$ National Synchrotron Radiation Laboratory, University of Science and Technology of China, Hefei 230026, China

Beijing National Laboratory for Condensed Matter Physics, Institute of Physics, Chinese Academy of Sciences, Beijing 100190, China

${ }^{\perp}$ Key Laboratory of Space Utilization, Technology and Engineering Center for Space Utilization, Chinese Academy of Sciences, Beijing 100094, China

"School of Electronic Science and Applied Physics, Hefei University of Technology, Hefei 230009, China

"Department of Physics, Beijing Normal University, Beijing 100875, China

$\nabla$ College of Science, National University of Defense Technology, Changsha 410073, China

\section{AUTHOR INFORMATION}

\section{Corresponding Authors}

*E-mail: zhangsen@nudt.edu.cn.

*E-mail: ygzhao@tsinghua.edu.cn. 


\section{fields}

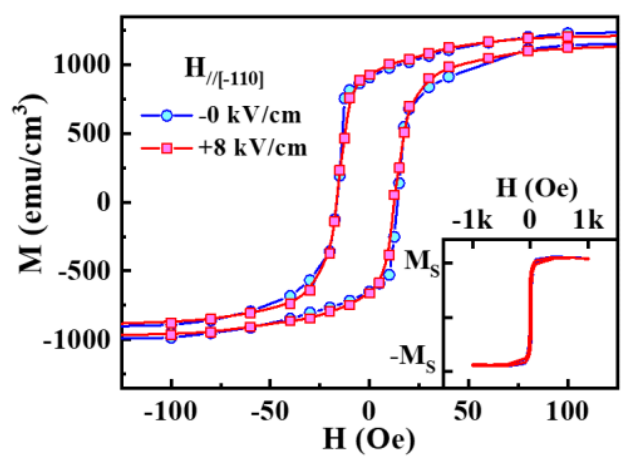

Figure S1. Magnetic hysteresis loops along the [-110] direction under electric fields of $-0 \mathrm{kV} / \mathrm{cm}$ (blue dot) and $+8 \mathrm{kV} / \mathrm{cm}$ (red square).

\section{S2. P-E loops measured with different ranges of electric field}

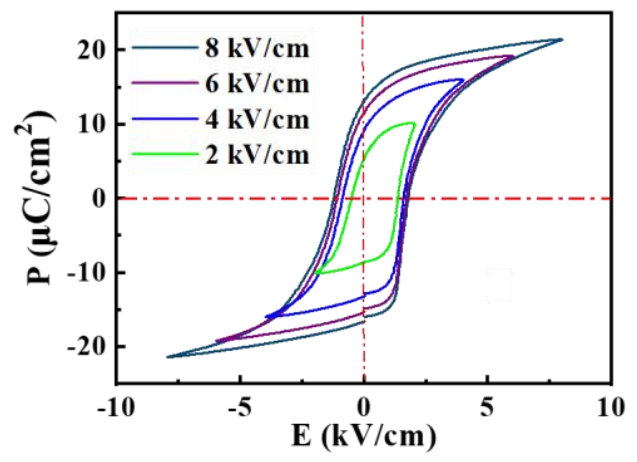

Figure S2. P-E loops measured with different ranges of electric field.

The P-E loops show an asymmetric polarization switching behavior for the positive and negative electric fields. A negatively biased internal electric field of $0.3 \mathrm{kV} / \mathrm{cm}$ can be deduced by the difference of positive and negative coercive electric fields. Moreover, it is easy to reach the saturation polarization under negative fields, while 
harder under positive ones (especially in the case of small electric-field range), suggesting the existence of phase transition induced by the positive electric field.

\section{S3. Magnetoelectric coupling coefficient}

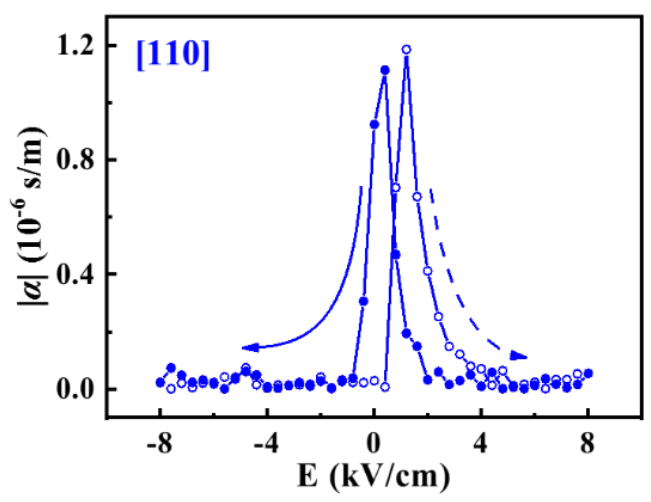

Figure S3. Magnetoelectric coupling coefficient $\left(\alpha=\mu_{0} \mathrm{~d} M / \mathrm{d} E\right)$ along the [110] direction deduced from Figure 1c. The arrows show the sweeping directions of electric field.

\section{S4. Raw ESR signals for some typical angles along the in-plane $\langle 110\rangle$ directions}
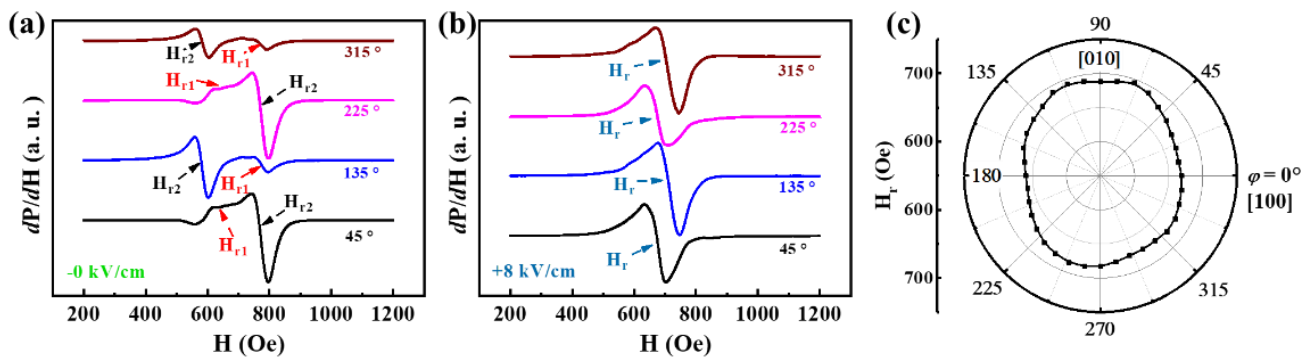

Figure S4. (a) The raw ESR data for some typical angles gathered along the in-plane $<110>$ directions for the case of $-0 \mathrm{kV} / \mathrm{cm}$. There are two peaks marked by $H_{\mathrm{r} 1}$ and $H_{\mathrm{r} 2}$, whose separation is maximum along the $\langle 110\rangle$ directions. (b) The raw ESR data for some typical angles along the in-plane $\langle 110\rangle$ directions for the case of $+8 \mathrm{kV} / \mathrm{cm}$. (c) 
In-plane $H_{\mathrm{r}}$ obtained for as-grown sample at various angles, marked with the black dots connected with a smooth line.

\section{S5. Kittel formula for fitting ESR data}

Kittel formula for ESR data with the uniaxial magnetic anisotropy model is as follows: ${ }^{1}$

$$
f=\frac{\gamma}{2 \pi} \sqrt{H_{r} \cos \left(\theta_{H}-\theta_{M}\right)+H_{K} \cos ^{2} \theta_{M}} \cdot \sqrt{H_{r} \cos \left(\theta_{H}-\theta_{M}\right)+H_{K} \cos 2 \theta_{M}}
$$

Where $f$ is the fixed radio frequency (Here $f=9.079 \mathrm{GHz}), H_{K}=2 K_{1} / M_{S}$ and $\gamma$ is the gyromagnetic ratio given by $\gamma=g \mu_{B} / \hbar$, where $g, \mu_{B}$, and $\hbar$ are Lande's $g$ factor, the Bohr magnetron and Plank's constant, respectively. $\theta_{\mathrm{H}}$ is defined as the angle between the applied magnetic field and the in-plane easy axis of magnetization, and $\theta_{\mathrm{M}}$ is the angle between the magnetic moment and the in-plane easy axis of magnetization. The saturation magnetization $\left(M_{\mathrm{S}}=1200 \mathrm{emu} / \mathrm{cm}^{3}\right.$ for $\left.\mathrm{CoFeB}\right)$ can be obtained by the magnetic hysteresis loops (Figure 1b). Using the FMR data, $H_{r}\left(\theta_{H}\right)$ can be obtained, and then, the parameters $K_{1}$ and $\mathrm{g}$ factor can be settled by fitting the $H_{\mathrm{r}} v s \theta_{\mathrm{H}}$ curves using the Kittel formula with the uniaxial magnetic anisotropy model.

The fitting gave $K_{1}=7.5 \times 10^{3} \mathrm{~J} / \mathrm{m}^{3}$ for $H_{\mathrm{r} 2}$ related to the main peak under -0 $\mathrm{kV} / \mathrm{cm}$, so that anisotropy field can be deduced as $H_{K}=2 K_{1} / M_{S}=125$ Oe . Via rotating the fitting line by $90^{\circ}$, it just matches the $H_{\mathrm{r} 1}$ related to the weaker peak, suggesting that it gives $K_{1}$ comparable to that deduced from $H_{\mathrm{r} 2}$. 
S6. XRD-RSM results and the analysis of polarization history under a sequence of electric fields

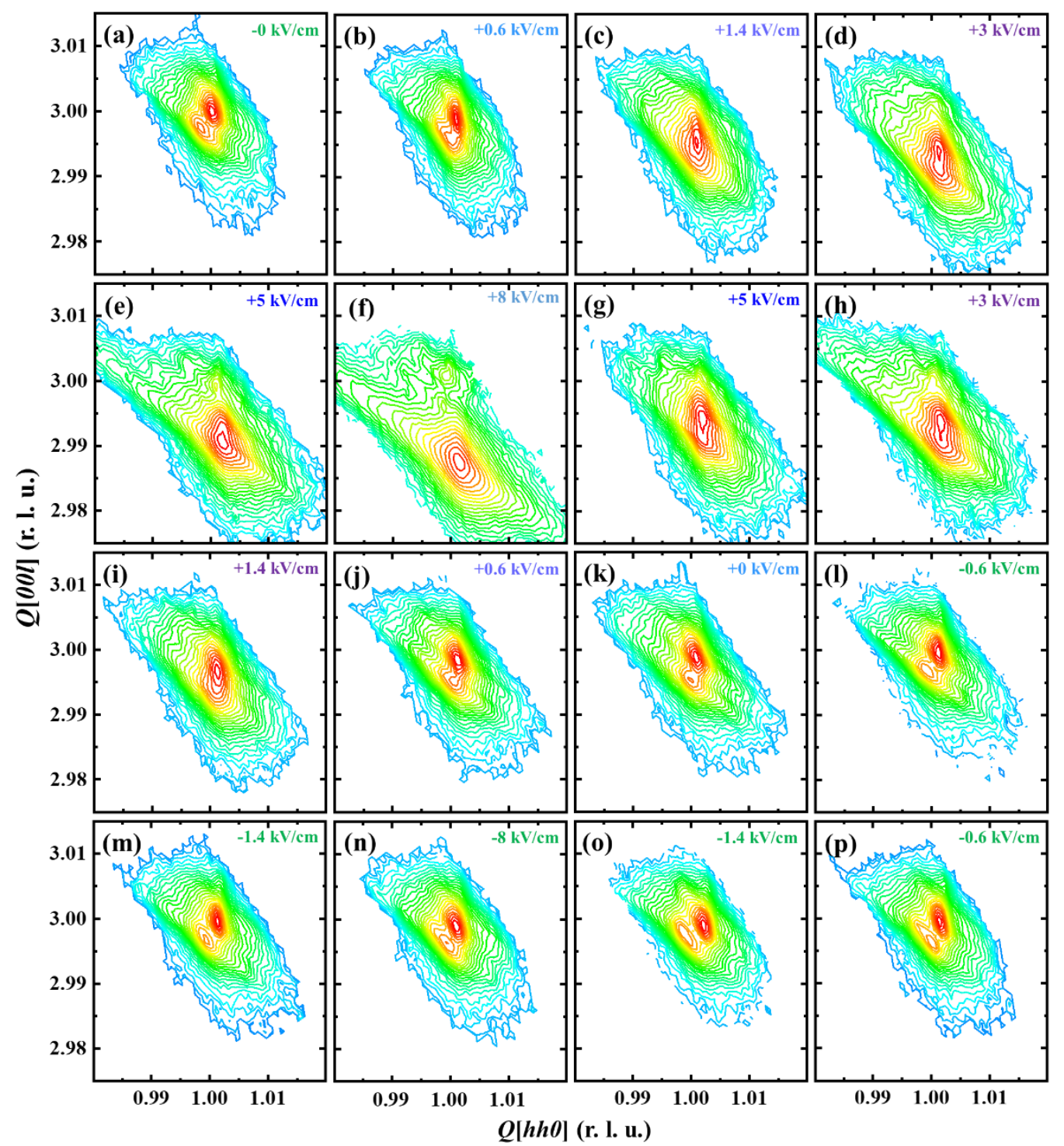

Figure S5. (a-p) XRD-RSM results around the (113) peak under a sequence of electric fields. 

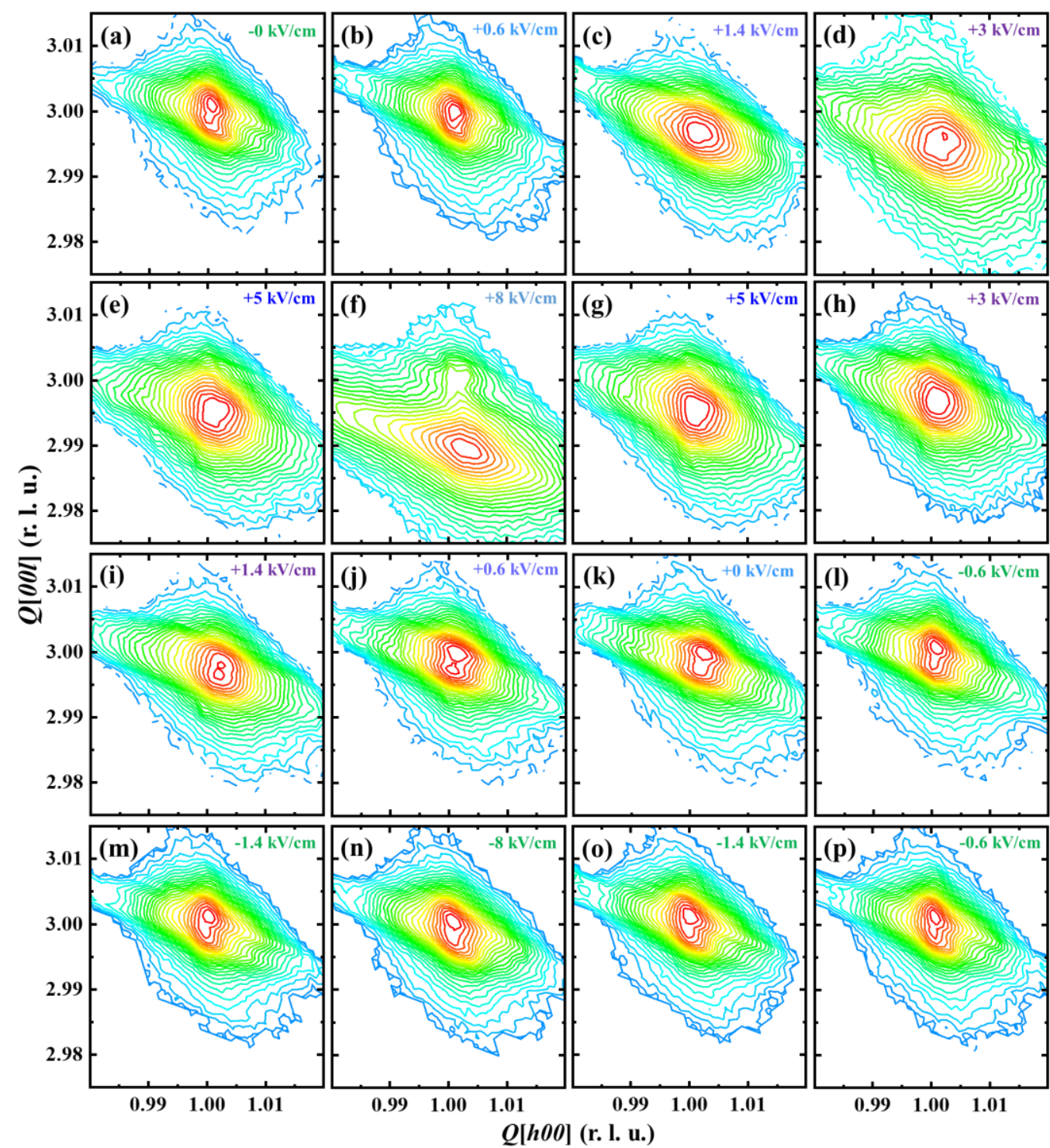

Figure S6. (a-p) XRD-RSM results around the (103) peak under a sequence of electric fields.

In PMN-PT crystals, the polarization and the crystal structure are coupled, so that we can detect the crystal structure under different electric field history by XRD-RSM and deduce the polarization history and corresponding phase transition. The polarization undergoes the process of switching and rotation in the present of bipolar electric field. The rotation process is relatively clear accompanied by the phase transition $\mathrm{M}_{\mathrm{A}}-\mathrm{T}$ and 
it is coherent rotation in light of the gradual change for the positions of diffraction spots in Figure S5d-i. However, the polarization switching process is fairly ambiguous due to the quite sharp change in XRD diffraction in Figure S5a-c and Figure S5k-m. The positions of diffraction in the polarization switching process cannot match a single pseudocubic crystal structure, so that we take this process as the nucleation and growth. ${ }^{2}$ In this way, we summarized the polarization history and corresponding phase transition in Figure S7.

(a)

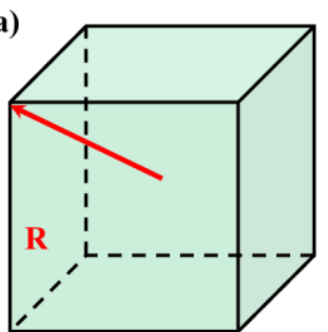

(b)

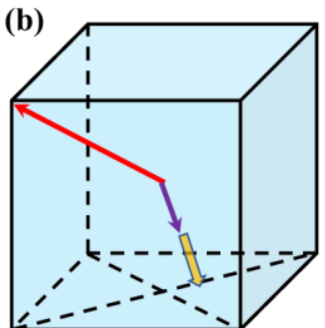

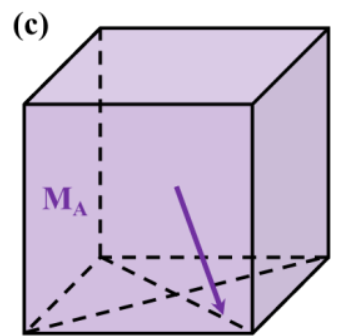

Nucleation and growth

(d)
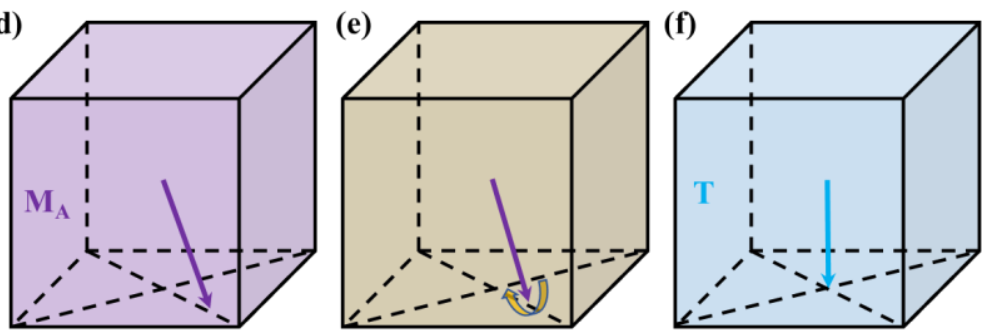

Coherent rotation

Figure S7. A typical polarization history. (a-c) the polarization switching process between $R$ and $M_{A}$ phase undergoing the nucleation and growth. (d-f) the polarization rotation process between $\mathrm{M}_{\mathrm{A}}$ and $\mathrm{T}$ phase undergoing coherent rotation.

\section{S7. XRD results around the (002) peak under a sequence of electric fields}



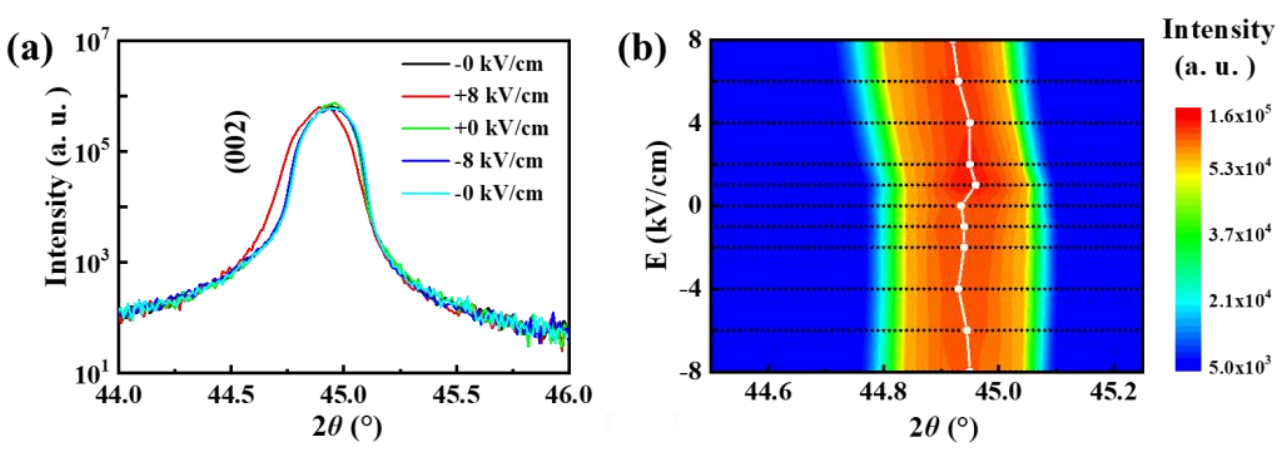

Figure S8. (a) $\theta-2 \theta$ scan around the (002) peak under different electric fields. (b)

Electric field $v s$ the angle of (002) peak with the black dots showing the data points and the white dots marking the angles of the peaks.

\section{S8. The ratio of various $R$ phase distortions and the in-plane strains under -0}

\section{$\mathbf{k V} / \mathbf{c m}$}
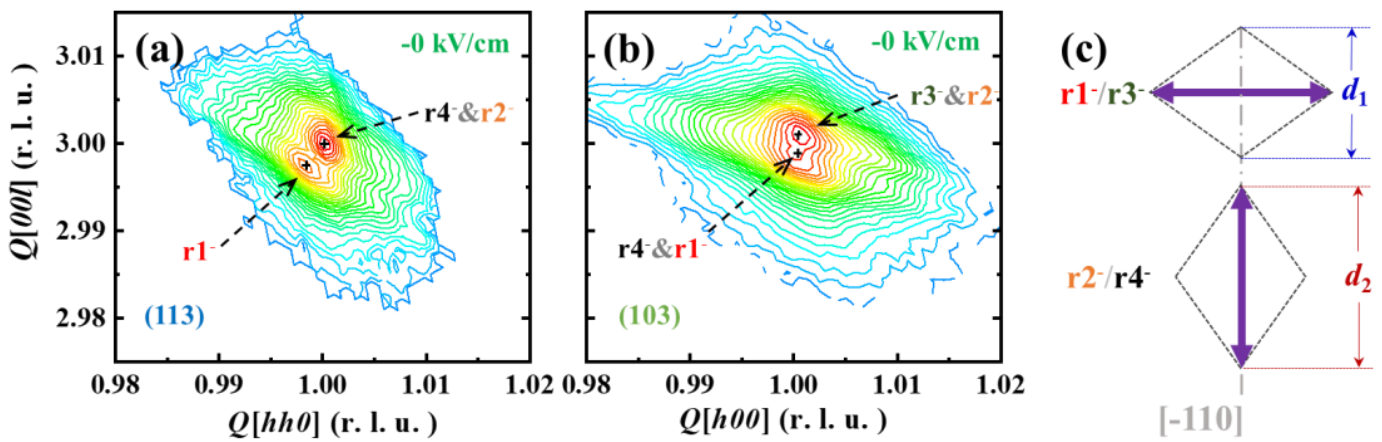

Figure S9. $(a, b)$ The RSM measurements to investigate the crystal structure distortions by the (113) and (103) reflections under $-0 \mathrm{kV} / \mathrm{cm}$. (c) The in-plane distortions of the R phase crystal structures.

Table S1. The positions and intensities of the (113) refection peaks for the negatively poled FE, with coordinate values defined by the cubic crystal structure (lattice parameter $a_{0}=0.403 \mathrm{~nm}$ ). The rough ratios of the $\mathrm{R}$ phase distortions can be 
computed easily by the peak intensity.

\begin{tabular}{ccccc}
\hline (113) & $\boldsymbol{Q}[\boldsymbol{h h 0 ]}$ & $\boldsymbol{Q}[\mathbf{0 0 l}]$ & intensity & ratio \\
\hline r1 $^{-}$ & $0.99817 \pm 0.00038$ & $2.99782 \pm 0.00070$ & 6402 & $1 / 3$ \\
$\mathrm{r}^{-} \& \mathrm{r}^{-}$ & $1.00031 \pm 0.00032$ & $3.00006 \pm 0.00082$ & 13481 & $2 / 3$ \\
r3 $^{-}$ & - & - & - & 0 \\
\hline
\end{tabular}

Table S2. The positions and intensities of the (103) refection peaks for the negatively poled FE.

\begin{tabular}{ccccc}
\hline $\mathbf{( 1 0 3 )}$ & $\boldsymbol{Q}[\boldsymbol{h 0 0}]$ & $\boldsymbol{Q}[\mathbf{0 0 l}]$ & intensity & ratio \\
\hline $\mathrm{r}^{-} \& \mathrm{rr}^{-}$ & $0.99987 \pm 0.00067$ & $2.99857 \pm 0.00065$ & 44190 & $2 / 5$ \\
$\mathrm{r}^{-} \& \mathrm{rr}^{-}$ & $1.00021 \pm 0.00076$ & $3.00075 \pm 0.00062$ & 69000 & $3 / 5$ \\
\hline
\end{tabular}

Table S3. The rough ratios of the four R phase distortions for the negatively poled FE.

\begin{tabular}{llllll}
\hline $\mathbf{- 0} \mathbf{~ k V / c m}$ & $\mathbf{r 1}^{-}$ & $\mathbf{r 2}^{-}$ & $\mathbf{r 3}^{-}$ & $\mathbf{r 4}^{-}$ \\
\hline & $33.3 \%$ & $60 \%$ & 0 & $6.7 \%$ \\
\hline
\end{tabular}

According to the positions of the (113) reflection peaks, the in-plane strains of the $\mathrm{R}$ phase distortions can be calculated:

$$
\varepsilon=\frac{d_{2}-d_{1}}{\sqrt{2} a_{0}}=\frac{1}{Q[h h 0]\left(r 1^{-}\right)}-\frac{1}{Q[h h 0]\left(r 2^{-} \& r 4^{-}\right)}=(0.22 \pm 0.07) \%
$$

Based on the ratios obtained by the reflection peak intensities around the (113) and (103), the ratios of the four $\mathrm{R}$ phase distortions for the negatively poled $\mathrm{FE}$ are listed in Table S3. 


\section{S9. Analysis of the in-plane strains under the sequence of electric fields}

Table S4. The peak positions in diffraction patterns around the (113) under the sequence of electric fields, with the coordinate values defined by the cubic crystal structure $\left(a_{0}=0.403 \mathrm{~nm}\right)$. There are usually two peaks in Figure S5, which are named "right" and "left" to distinguish them, and their coordinate values (include the axes of $Q[h h 0]$ and $Q[00 l])$ under the sequence of electric fields can be obtained.

\begin{tabular}{|c|c|c|c|c|c|c|c|c|}
\hline $\begin{array}{c}E \\
(\mathrm{kV} / \mathrm{cm})\end{array}$ & $\begin{array}{l}Q[h h 0] \\
\text { (right) }\end{array}$ & $\begin{array}{c}\delta Q[h h 0] \\
\text { (right) }\end{array}$ & $\begin{array}{c}Q[h h 0] \\
\text { (left) }\end{array}$ & $\begin{array}{c}\delta Q[h h 0] \\
\text { (left) }\end{array}$ & $\begin{array}{c}Q[00 l] \\
\text { (right) }\end{array}$ & $\begin{array}{c}\delta Q[00 l] \\
\text { (right) }\end{array}$ & $\begin{array}{c}Q[00 l] \\
\text { (left) }\end{array}$ & $\begin{array}{c}\delta Q[00 l] \\
\text { (left) }\end{array}$ \\
\hline 0 & 1.00031 & 0.00032 & 0.99817 & 0.00038 & 3.00006 & 0.00082 & 2.99782 & 0.00070 \\
\hline 0.6 & 1.00084 & 0.00010 & 0.99933 & 0.00020 & 2.99893 & 0.00050 & 2.99643 & 0.00040 \\
\hline 1.4 & 1.00081 & 0.00030 & 1.00081 & 0.00030 & 2.99544 & 0.00040 & 2.99544 & 0.00040 \\
\hline 3 & 1.00112 & 0.00040 & 1.00122 & 0.00040 & 2.99367 & 0.00030 & 2.99125 & 0.00030 \\
\hline 5 & 1.00195 & 0.00040 & 1.00195 & 0.00040 & 2.99076 & 0.00030 & 2.99076 & 0.00030 \\
\hline 8 & 1.00133 & 0.00040 & 1.00133 & 0.00040 & 2.98753 & 0.00040 & 2.98753 & 0.00040 \\
\hline 5 & 1.00185 & 0.00040 & 1.00185 & 0.00040 & 2.99448 & 0.00030 & 2.99448 & 0.00030 \\
\hline 3 & 1.00170 & 0.00030 & 1.00158 & 0.00040 & 2.99405 & 0.00040 & 2.99101 & 0.00030 \\
\hline 1.4 & 1.00123 & 0.00020 & 1.00123 & 0.00020 & 2.99672 & 0.00040 & 2.99672 & 0.00040 \\
\hline 0.6 & 1.00092 & 0.00030 & 1.00023 & 0.00020 & 2.99849 & 0.00030 & 2.99506 & 0.00030 \\
\hline 0 & 1.00084 & 0.00030 & 0.99986 & 0.00030 & 2.99893 & 0.00040 & 2.99530 & 0.00030 \\
\hline-0.6 & 1.00134 & 0.00030 & 0.99936 & 0.00040 & 2.99976 & 0.00020 & 2.99688 & 0.00040 \\
\hline-1.4 & 1.00135 & 0.00020 & 0.99925 & 0.00040 & 2.99976 & 0.00030 & 2.99688 & 0.00040 \\
\hline-8 & 1.00084 & 0.00030 & 0.99933 & 0.00040 & 2.99893 & 0.00030 & 2.99642 & 0.00040 \\
\hline-1.4 & 1.00202 & 0.00020 & 0.99881 & 0.00030 & 2.99926 & 0.00030 & 2.99782 & 0.00020 \\
\hline-0.6 & 1.00135 & 0.00030 & 0.99995 & 0.00020 & 2.99976 & 0.00040 & 2.99681 & 0.00030 \\
\hline 0 & 1.00031 & 0.00032 & 0.99817 & 0.00038 & 3.00006 & 0.00082 & 2.99782 & 0.00070 \\
\hline
\end{tabular}



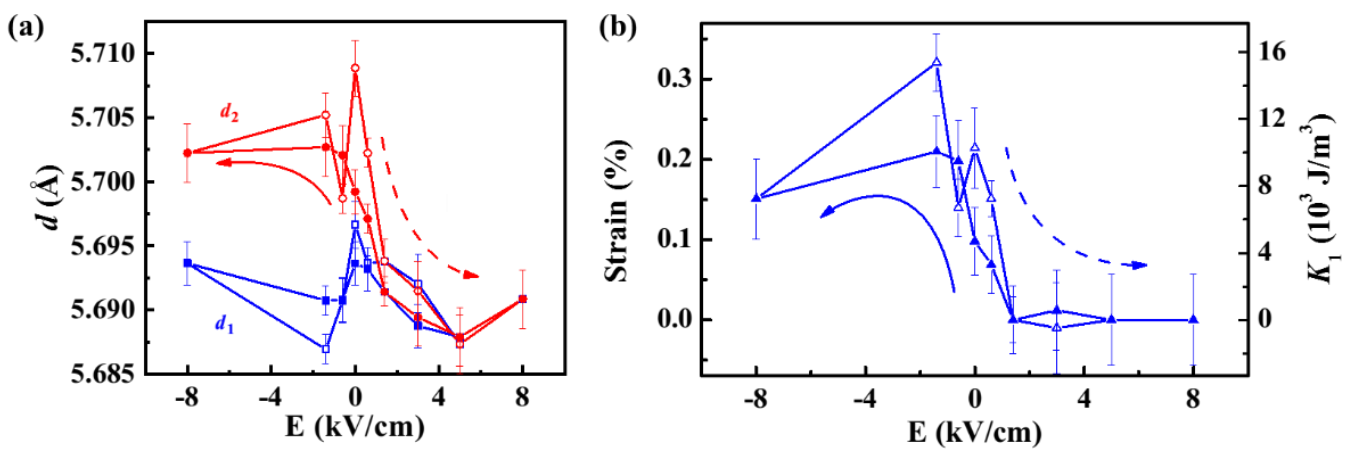

Figure S10. (a) $d_{1}, d_{2}$, calculated in the similar way as the case showing before (Supporting Information S8), change with electric field and show hysteresis in the range between $-0.3 \mathrm{kV} / \mathrm{cm}$ and $+1.4 \mathrm{kV} / \mathrm{cm}$ with arrows guiding the sweeping directions of the curves. (b) strain along the elongated direction in each domain calculated by $\left(d_{2}-d_{1}\right) / \sqrt{2} a_{0}$, and Magnetic anisotropic constant $K_{1}$ obtained through the calculation by the strain-induced anisotropy energy as mentioned in the main text of the paper.

There is hysteresis for magnetic anisotropic constant $K_{1}$ with fields between -0.3 $\mathrm{kV} / \mathrm{cm}$ and $+3 \mathrm{kV} / \mathrm{cm}$, which can bring out the hysteresis in the M-E curves (Figure 1c). However, the difference of $K_{1}$ under $-1.4 \mathrm{kV} / \mathrm{cm}$ cannot induce hysteresis in the M-E curves because the equivalent magnetic fields (257 Oe and 168 Oe for the case of increasing and decreasing magnetic fields, respectively) related to $K_{1}$ in this case are much larger than the 5 Oe external magnetic field contributing to the Zeeman energy, so that the magnetic moment still remains along the direction of the equivalent magnetic field. 
S10. $M_{A}$ phase distortion model and phase transition induced by the sequence of electric fields

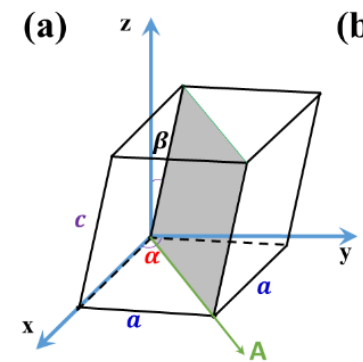

(b)

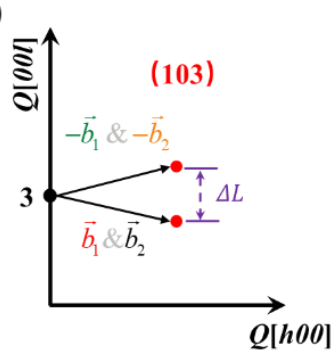

(c)

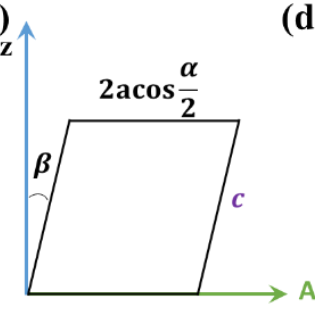

(d)

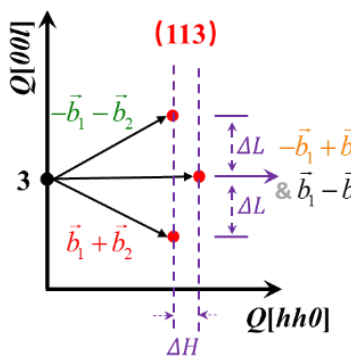

(e)

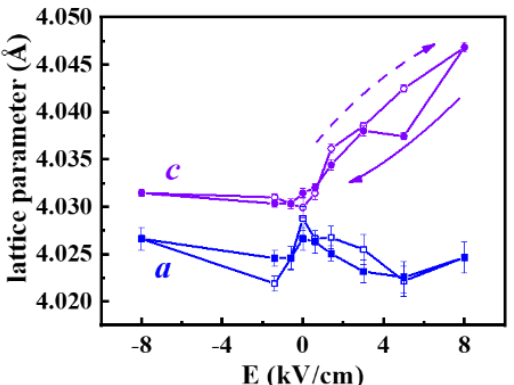

(f)

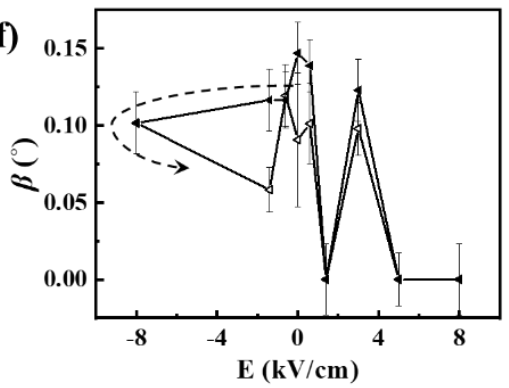

Figure S11. (a) $\mathrm{M}_{\mathrm{A}}$ phase distortion (including the $\mathrm{R}$ and $\mathrm{T}$ phase distortions as the special cases) is described by lattice parameters ( $a$ and $c$ ) and angles $(\alpha$ and $\beta$ ). The section filled by grey is in the Az plane shown in (c). (b,d) The positions of (103) and (113) diffraction spots related to different distortions. $(\mathrm{e}, \mathrm{f})$ The lattice parameters $(a$ and $c$ ) and angle $\beta$ vary under the sequence of electric fields.

Let $\vec{a}_{1}, \vec{a}_{2}$, and $\vec{a}_{3}$ be the set of primitive vectors for the direct lattice in $\mathrm{M}_{\mathrm{A}}$ phase distortion shown in Figure S11a.

$$
\begin{gathered}
\vec{a}_{1}=(a, 0,0) \\
\vec{a}_{2}=(a \cos \alpha, a \sin \alpha, 0) \\
\vec{a}_{3}=\left(c \sin \beta \cos \frac{\alpha}{2}, c \sin \beta \sin \frac{\alpha}{2}, c \cos \beta\right)
\end{gathered}
$$

Then the reciprocal lattice can be generated by the three primitive vectors. 


$$
\begin{gathered}
\overrightarrow{\mathrm{b}}_{1}=\frac{\vec{a}_{2} \times \vec{a}_{3}}{\vec{a}_{1} \cdot\left(\vec{a}_{2} \times \vec{a}_{3}\right)}=\frac{1}{a}\left(1,-\frac{1}{\tan \alpha},-\frac{\tan \beta}{2 \cos \frac{\alpha}{2}}\right) \\
\vec{b}_{2}=\frac{\vec{a}_{3} \times \vec{a}_{1}}{\vec{a}_{1} \cdot\left(\vec{a}_{2} \times \vec{a}_{3}\right)}=\frac{1}{a}\left(0, \frac{1}{\sin \alpha},-\frac{\tan \beta}{2 \cos \frac{\alpha}{2}}\right) \\
\vec{b}_{3}=\frac{\vec{a}_{1} \times \vec{a}_{2}}{\vec{a}_{1} \cdot\left(\vec{a}_{2} \times \vec{a}_{3}\right)}=\frac{1}{c}\left(0,0, \frac{1}{\cos \beta}\right)
\end{gathered}
$$

Using the first-order approximation of angles $(\alpha$ and $\beta)$, the distribution of the diffraction spots around the (103) and (113) is exhibited in Figure S11c,d, respectively, ${ }^{3}$ with the parameters $Q[h h 0]($ left), $\Delta \mathrm{H}, \Delta \mathrm{L}$ and the $Q[00 l]$ (right) calculated as:

$$
\begin{gathered}
\frac{\sqrt{2}}{a_{0}} Q[\text { hhO }](\text { left })=\frac{\sqrt{2}}{a} \\
\frac{\sqrt{2}}{a_{0}} \Delta H=\frac{\sqrt{2}}{2 a}\left(1+\frac{1}{\sin \alpha}+\frac{1}{\tan \alpha}\right)-\frac{\sqrt{2}}{2 a}\left(1+\frac{1}{\sin \alpha}-\frac{1}{\tan \alpha}\right)=\frac{\sqrt{2}}{a \tan \alpha}=\frac{\sqrt{2}}{a}\left(\frac{\pi}{2}-\alpha\right) \\
\frac{1}{a_{0}} \Delta L=\frac{1}{a}\left(2 \times \frac{\tan \beta}{2 \cos \frac{\alpha}{2}}\right)=\frac{\sqrt{2}}{2} \beta * \frac{2}{a}=\frac{\sqrt{2} \beta}{a} \\
\frac{1}{a_{0}} Q[00 l](\text { right })=\frac{3}{c \cos \beta}
\end{gathered}
$$

So that we can calculate the lattice parameters and angles using the data in Table S4. It needs to be mentioned that there are just two spots in the measurement of the (113) reflections at most rather than three spots expected from analysis (Figure S11d), which was also exhibited in the RSM results on PMN-xPT crystals in the previous report $^{4}$ owing to the inhomogeneity of spontaneous polarization. Therefore, the right peaks around the (113), marked as $\mathrm{a}_{2}$ and $\mathrm{a}_{4}$ in $\mathrm{M}_{\mathrm{A}}$ phase distortions, are always 
$\left(\vec{b}_{1}-\vec{b}_{2}\right) \&\left(-\vec{b}_{1}+\vec{b}_{2}\right)$, deviated from the (003) spots. The left ones, marked as a in $\mathrm{M}_{\mathrm{A}}$ phase distortions, are $\left(\vec{b}_{1}+\vec{b}_{2}\right)$, deviated from the (003) spots.

In addition, it was mentioned before that this model includes the $\mathrm{R}$ and $\mathrm{T}$ phase distortions as the special cases, and we can distinguish the special cases of the $\mathrm{R}$ and $\mathrm{T}$ phase distortions by the equations shown below.

For the R phase distortion, $\cos \alpha=\sin \beta \cos \frac{\alpha}{2}, a=c$

For the T phase distortion, $\alpha=90^{\circ}, \beta=0, a \neq c$

On account of the large error in the analysis of $\beta$, other equations without $\beta$ become the conditions to distinguish the $\mathrm{R}, \mathrm{M}_{\mathrm{A}}$ and $\mathrm{T}$ distortions. The $\mathrm{R}$ phase distortions appear under negative electric fields with the feature of close values for $a$ and $c$. The $\mathrm{T}$ phase distortion appears when electric field reaches $+5 \mathrm{kV} / \mathrm{cm}$ with the feature of $\alpha=90^{\circ}$. The polarization of $\mathrm{M}_{\mathrm{A}}$ distortions between $\mathrm{R}$ and $\mathrm{T}$ phases can rotate under various electric fields, which can be revealed by the change of $\alpha$. 
S11. FE domain switching in the PMN-0.18PT measured with PFM
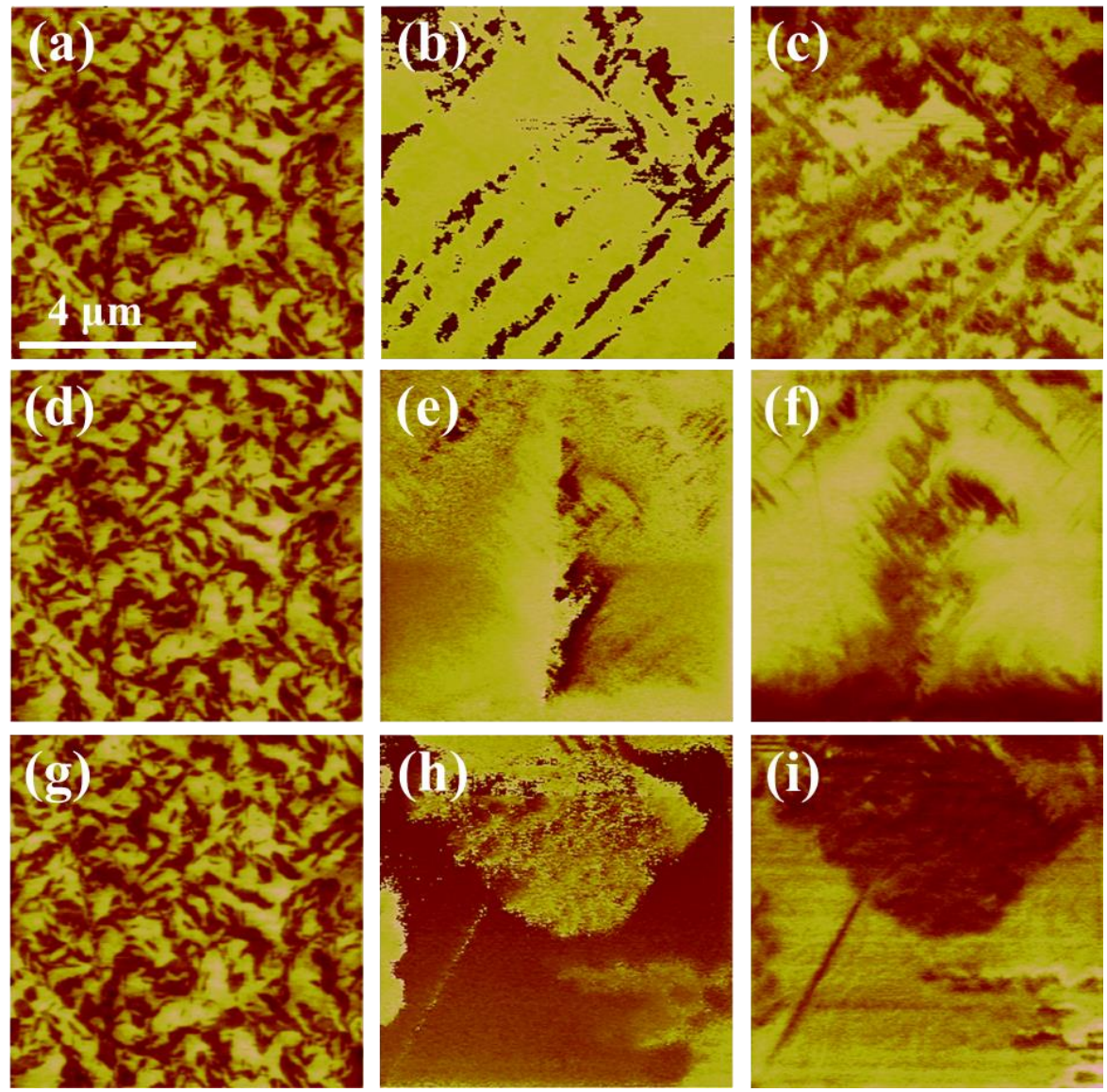

Figure S12. $(a, d, g)$ The topography images. $(b, e, h)$ The out-of-plane phase PFM images of the as-grown PMN-0.18PT and after poled by the PFM tip with in situ +10 $\mathrm{V}$ and $-10 \mathrm{~V}$ dc voltage, respectively. (c,f,i) The in-plane phase PFM images of the PMN-0.18PT in the three states, respectively. 
S12. Schematic diagram of the FE, FM domains and effect of a 5 Oe magnetic field
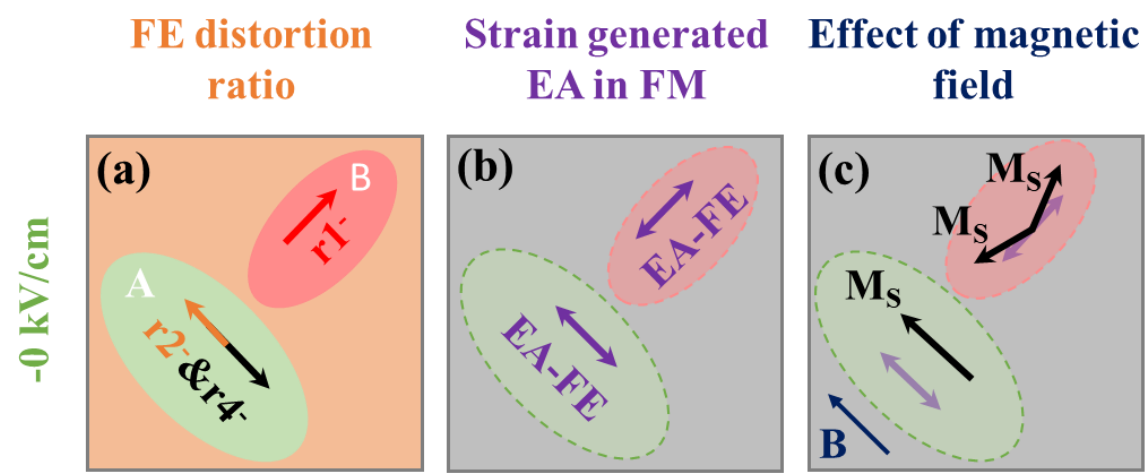

\section{EA in FM}
field
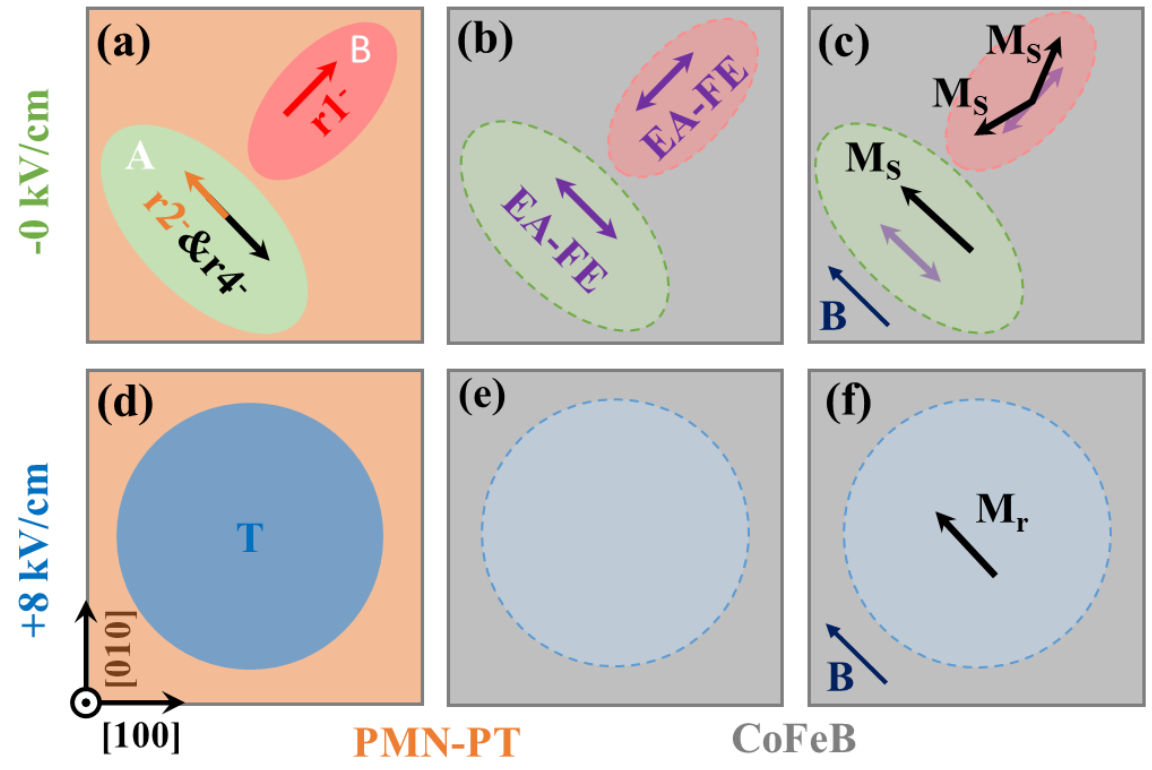

Figure S13. Schematic for both FE domains and the corresponding FM domains of CoFeB/PMN-0.18PT under different electric fields. (a,d) shows the FE domain A and domain B with ellipses (large ellipse represents a large portion) and the arrows are the in-plane polarization directions for the case of $-0 \mathrm{kV} / \mathrm{cm}$ and $\mathrm{FE}$ domain with only out-of-plane polarization for the case of $+8 \mathrm{kV} / \mathrm{cm}$, respectively. (b,e) Illustrations for domain coupling of FE and FM layers under different electric fields. The black double-headed arrow (EA-FE) indicates the magnetic easy axis of FM induced by the in-plane strain in the FE domains. (c,f) The directions of magnetization in the FM layer upon applying a weak magnetic field along the [-110], for example, the remnant magnetization is marked as $M_{\mathrm{S}}(M \mathrm{r})$ without (with) the absence of magnetic anisotropy. 
As shown in Figure S13a, the large ellipse is FE domain A with r2 $^{-} \& 4^{-}$polarization, while the small one is the FE domain $\mathrm{B}$ with $\mathrm{r1}^{-}$polarization. As a result, two kinds of FM domains appear due to the ME coupling between FE domains and FM domains with the uniaxial easy axes of magnetization (EA-FE) on the axes of [110] and [-110], respectively, as shown in Figure S13b. Figure S13c shows that with a 5 Oe in-plane magnetic field as used in the measurements of M-E curves, along the [-110] for example, the magnetization $\left(2 / 3 M_{\mathrm{S}}\right)$ is mainly contributed by the domain A whose magnetic easy axis is on the axis of [-110], while the magnetization in the domain B only tilts a little bit since the equivalent magnetic field (125 Oe, Supporting Information S5) related to the uniaxial magnetic anisotropy is much larger than 5 Oe. However, for the case of $+8 \mathrm{kV} / \mathrm{cm}$, the electric-field-induced $\mathrm{T}$ phase has an in-plane isotropic strain state as shown in Figure S13d, which leads to the in-plane magnetic isotropy in FM film, (Figure S13e), so that the magnetization will respond to the $5 \mathrm{Oe}$ magnetic fields more easily as shown in Figure S13f. It should be mentioned that the remnant magnetization for the FM domains along the magnetic easy axis for the case of $-0 \mathrm{kV} / \mathrm{cm}$ will be close to the saturation magnetization $M_{\mathrm{S}}$, larger than the remnant magnetization marked as $M_{\mathrm{r}}$ for the case of $+8 \mathrm{kV} / \mathrm{cm}$ as discussed later.

\section{S13. Comparison of $M s$ and $M r$}

Figure S1 shows that the remnant magnetization is somewhat maintained along the

[-110] direction when the sample was applied electric fields of $-0 \mathrm{kV} / \mathrm{cm}$ and +8 
$\mathrm{kV} / \mathrm{cm}$, marked as $M_{\mathrm{r}-}$ and $M_{\mathrm{r}+}$, respectively. It can be seen that $M_{\mathrm{r}-} \approx M_{\mathrm{r}^{+}}$. As mentioned in the main text, the remnant magnetization for the FM domains along the magnetic easy axis for the case of $-0 \mathrm{kV} / \mathrm{cm}$ is close to the saturation magnetization $M_{\mathrm{S}}$. Noting that only around two thirds of the total magnetization along the [-110] directions under $-0 \mathrm{kV} / \mathrm{cm}$ without a magnetic field, thus the $M_{\mathrm{r}}$ is about $2 / 3 M_{\mathrm{S}}$. While, for the case of $+8 \mathrm{kV} / \mathrm{cm}$, it shows magnetic isotropic and $M_{\mathrm{r}+}$ is $M_{\mathrm{r}}$. Since $M_{\mathrm{r}-}$ $\approx M_{\mathrm{r}+}$ and $M_{\mathrm{r}-} \approx 2 / 3 M_{\mathrm{S}}$, it can be deduced that $M_{\mathrm{r}} \approx 0.67 M_{\mathrm{S}}$.

S14. Magnetization Calculations along the [100] direction under $-0 \mathrm{kV} / \mathrm{cm}$ and +8 $\mathbf{k V} / \mathbf{c m}$
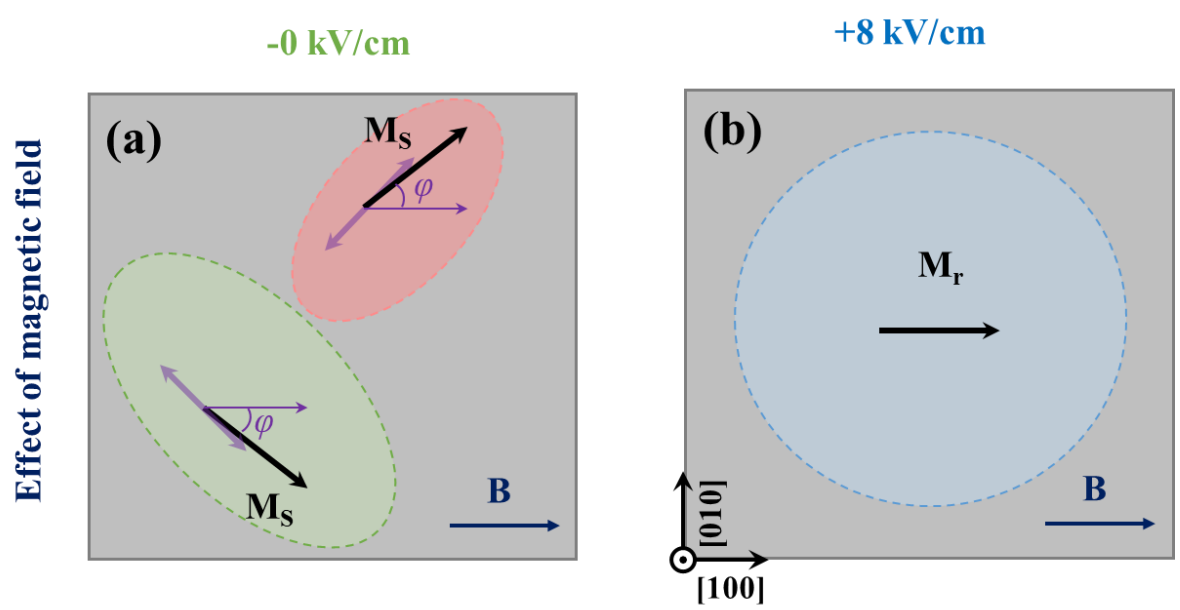

Figure S14. $(a, b)$ The directions of magnetization in the FM layer under a weak magnetic field along the [100], and the magnetization is marked as $M_{\mathrm{S}}\left(M_{\mathrm{r}}\right)$ with (without) the magnetic anisotropy under $-0 \mathrm{kV} / \mathrm{cm}(+8 \mathrm{kV} / \mathrm{cm})$.

Both the directions of the magnetization measurement and the weak magnetic field are along the [100], so the magnetization in this direction under $+8 \mathrm{kV} / \mathrm{cm}$ is $M_{\mathrm{r}}$ 
without the magnetic anisotropy. However, for the case of $-0 \mathrm{kV} / \mathrm{cm}$, the measured magnetization is the projections of the magnetizations in two kinds of FM domains with perpendicular magnetic easy axes but the same magnetic anisotropy energy. In this case, we need to calculate $\cos \varphi$ first. For each kind of FM domains, the free energy density satisfies the following formula and its minimal value can be obtained by $\frac{\partial f}{\partial \varphi}=0$.

$$
f=K_{1} \sin ^{2}\left(\frac{\pi}{4}-\varphi\right)-M_{S} H \cos \varphi
$$

Where $\quad M_{S} H=1200 \mathrm{emu} / \mathrm{cm}^{3} \times 5 \mathrm{Oe}=6000 \mathrm{erg} / \mathrm{cm}^{3}=600 \mathrm{~J} / \mathrm{m}^{3} \quad, \quad$ and $K_{1}=7.5 \times 10^{3} \mathrm{~J} / \mathrm{m}^{3}$, so that we can calculate that $\cos \varphi=0.7263$. Therefore, the measured magnetization along the [100] direction under $-0 \mathrm{kV} / \mathrm{cm}$ is $M_{[100]}=\frac{1}{3} M_{S} \cos \varphi+\frac{2}{3} M_{S} \cos \varphi=0.726 M_{S}$. 
S15. M-E curves and RSM measurements on samples of type II and type III
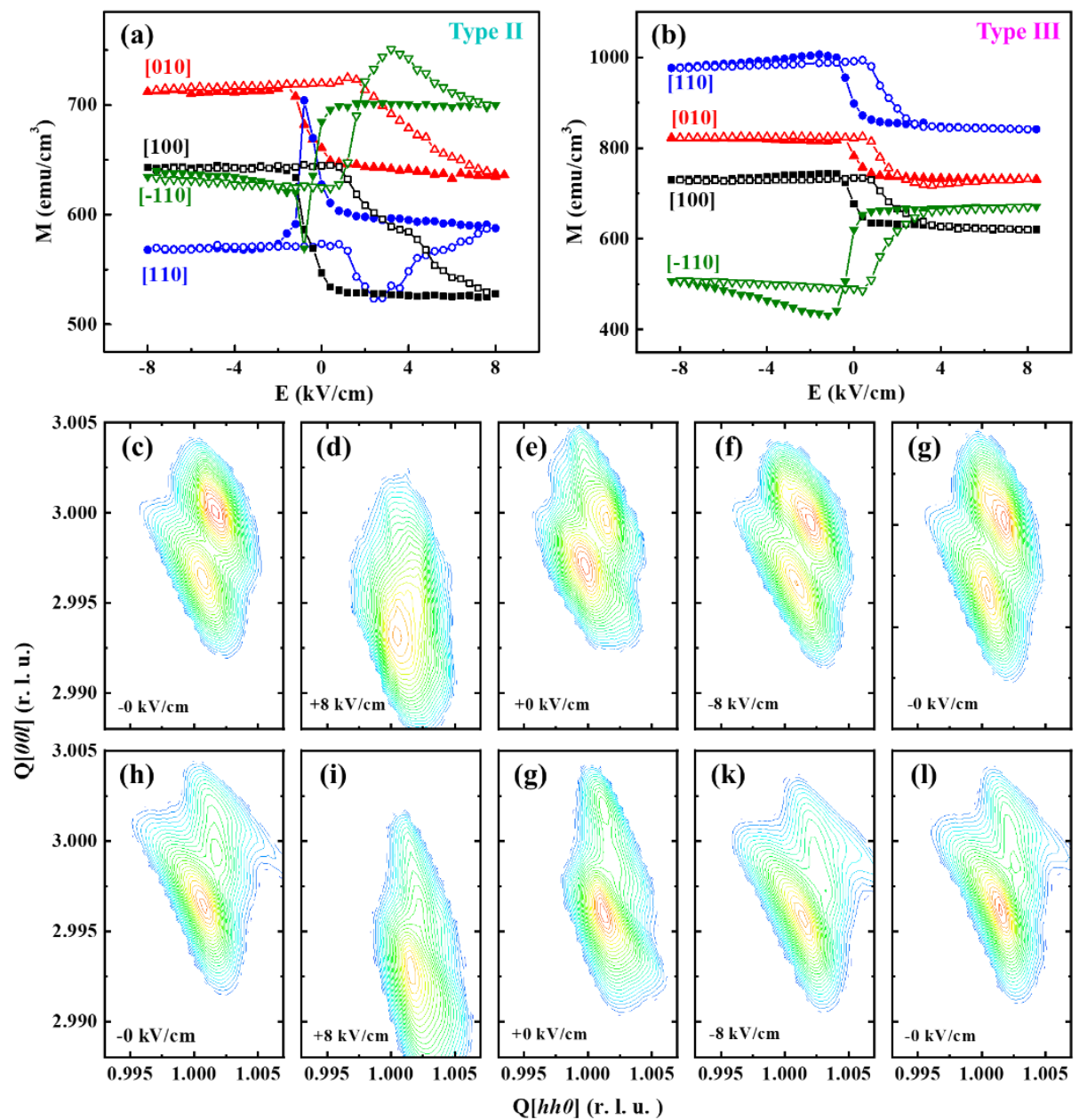

Figure S15. (a,b) Type II and type III M-E curves for samples 2 and 3 along the [010], [-110], [100] and [110] directions, respectively. (c-g) XRD-RSM results around the (113) peak under a sequence of electric fields for sample 2 . The phase transition to the T phase with $+8 \mathrm{kV} / \mathrm{cm}$ and $\xi$ around 0.44 can be deduced by the positions and intensities of diffraction spots. (h-l) XRD-RSM results around the (113) peak under a sequence of electric fields for sample 3, from which we can deduce the phase transition to the $\mathrm{T}$ phase with $+8 \mathrm{kV} / \mathrm{cm}$ and $\xi$ around 0.85 .

\section{S16. Determination of composition for the PMN-xPT crystal}




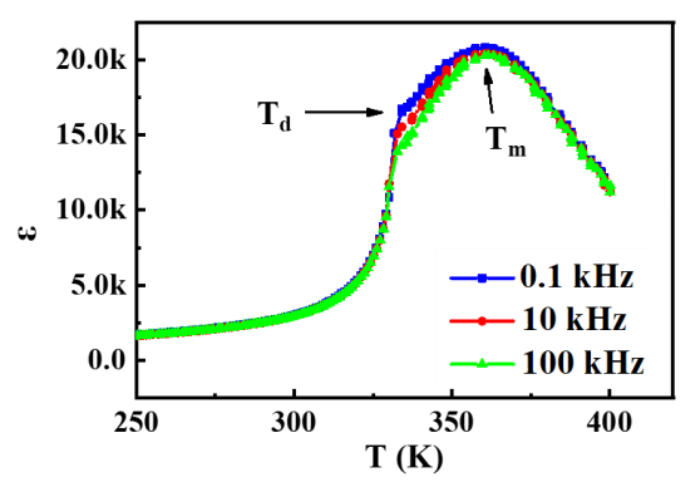

Figure S16. Variation of dielectric constant with temperature.

For PMN-xPT, there is a correlation between Curie temperature $T_{\mathrm{C}}$ and composition $\mathrm{x}$ as shown by Ye et al., ${ }^{5}$ so that we can use it to determine the composition of FE substrate through measuring $T_{\mathrm{C}}$. The $T_{\mathrm{C}}$ value can be obtained by averaging two temperatures, $T_{\mathrm{m}}$ (maximum) and $T_{\mathrm{d}}$ (depoling) obtained from dielectric measurements as shown by Noheda et al. ${ }^{6}$. We got $T_{\mathrm{d}}=332 \mathrm{~K}$ and $T_{\mathrm{m}}=360 \mathrm{~K}$ (Figure S16) from the variation of dielectric constant with temperature for the three frequencies, so that $T_{\mathrm{C}}=346 \mathrm{~K}$, which corresponds to $\mathrm{x}=0.18$ according to the correlation between Curie temperature $T_{\mathrm{C}}$ and composition $\mathrm{x}$.

\section{REFERENCES}

(1) He, W.; Liu, H. L.; Wu, H. Y.; Cai, J. W.; Cheng, Z. H. Probing Temperature-Driven Spin Reorientation Transition of GdFeCo Film by Kerr Loops and Ferromagnetic Resonance. Appl. Phys. Lett. 2015, 106, 042401.

(2) Dawber, M.; Rabe, K. M.; Scott, J. F. Physics of Thin-Film Ferroelectric Oxides. Rev. Mod. Phys. 2005, 77, 1083-1130.

(3) Christen, H. M.; Nam, J. H.; Kim, H. S.; Hatt, A. J.; Spaldin, N. A. 
Stress-Induced $R-M_{A}-M_{C}-T$ Symmetry Changes in $\mathrm{BiFeO}_{3}$ Films. Phys. Rev. $B$ 2011, 83, 144107.

(4) Zhang, S.; Zhao, Y. G.; Li, P. S.; Yang, J. J.; Rizwan, S.; Zhang, J. X.; Seidel, J.; Qu, T. L.; Yang, Y. J.; Luo, Z. L.; He, Q.; Zou, T.; Chen, Q. P.; Wang, J. W.; Yang, L. F.; Sun, Y.; Wu, Y. Z.; Xiao. X.; Jin, X. F.; Huang, J.; Gao, C.; Han, X. F.; Ramesh, R. Electric-Field Control of Nonvolatile Magnetization in $\mathrm{Co}_{40} \mathrm{Fe}_{40} \mathrm{~B}_{20} / \mathrm{Pb}\left(\mathrm{Mg}_{1 / 3} \mathrm{Nb}_{2 / 3}\right)_{0.7} \mathrm{Ti}_{0.3} \mathrm{O}_{3}$ Structure at Room Temperature. Phys. Rev. Lett. 2012, 108,137203.

(5) Ye, Z.-G.; Bing, Y.; Gao, J.; Bokov, A. A.; Stephens, P.; Noheda, B.; Shirane, G. Development of Ferroelectric Order in Relaxor $(1-x) \mathrm{Pb}\left(\mathrm{Mg}_{1 / 3} \mathrm{Nb}_{2 / 3}\right) \mathrm{O}_{3}-x \mathrm{PbTiO}_{3}$ $(0<=x<=0.15)$. Phys. Rev. B 2003, 67, 104104.

(6) Noheda, B.; Cox, D. E.; Shirane, G.; Gao, J.; Ye, Z.-G. Phase Diagram of the Ferroelectric Relaxor $(1-x) \mathrm{PbMg}_{1 / 3} \mathrm{Nb}_{2 / 3} \mathrm{O}_{3}-x \mathrm{PbTiO}_{3}$. Phys. Rev. B 2002, 66, 054104. 